\title{
Avaliação da oferta e da produção das especialidades odontológicas em serviços públicos de atenção secundária em um estado do nordeste brasileiro
}

\author{
Evaluation of the supply and production of dental specialties in public secondary care services in a
} state in northeastern Brazil

Evaluación de la oferta y producción de especialidades odontológicas en servicios públicos de atención secundaria en un estado del noreste de Brasil

\section{Resumo}

No Ceará a expansão da oferta dos Centros de especialidades odontológicas (CEO) é recente. Deste modo, o presente estudo teve como objetivo avaliar a oferta e a produção dos CEO do Ceará no período de 2008 a 2014 a partir de dados secundários registrados no Sistema de Informação do Sistema Único de Saúde. Os dados foram expressos em forma absoluta e percentual e utilizaram-se os testes do qui-quadrado e a correlação de Spearman. Os resultados mostraram que o número de procedimentos no ano de 2008 foi de 280222 (9.2\%), havendo uma leve redução para $253370(8.3 \%)$ em 2009, um aumento para 677664 (22.3\%) em 2010, com quedas subsequentes em $2011(\mathrm{n}=525259,17.3 \%), 2012(\mathrm{n}=430991, \mathrm{n}=14.2 \%)$ e $2013(\mathrm{n}=291666,9.6 \%)$ reassumindo crescimento em 2014 $(\mathrm{n}=457192,15.1 \%)$, totalizando 3035626 procedimentos no período de 2008-2014. Não houve correlação estatisticamente significante do número de procedimentos com o tempo do período avaliado (p=0.444). Em relação ao tipo de gestão os CEO municipais, $\mathrm{p}=0.003, \mathrm{r}=0.964$ e os CEO estaduais, $\mathrm{p}=0.048, \mathrm{r}=0.775$ ) apresentaram crescimento significante no período avaliado. Apesar da expansão dos serviços de saúde bucal secundária no Ceará e do crescente número de procedimentos especializados registrados, a capacidade para resolver os problemas destes serviços ainda é considerada um desafio para os gestores e profissionais da saúde bucal.

Palavras-chave: Saúde bucal; Avaliação de serviços de saúde; Especialidades odontológicas.

\begin{abstract}
In Ceará, the expansion of the offer of the Dental Specialties Centers (CEO) is recent. Thus, the present study aimed to evaluate the supply and production of CEOs from Ceará in the period from 2008 to 2014 from secondary data recorded in the Information System of the Unified Health System. The data were expressed in absolute and percentage form and the chi-square tests and Spearman's correlation were used. The results showed that the number of procedures in 2008 was $280222(9.2 \%)$, with a slight reduction to $253370(8.3 \%)$ in 2009, an increase to $677664(22.3 \%)$ in 2010, with subsequent falls in $2011(\mathrm{n}=525259,17.3 \%), 2012(\mathrm{n}=430991, \mathrm{n}=14.2 \%)$ and $2013(\mathrm{n}=291666,9.6 \%)$ resuming growth in $2014(\mathrm{n}=457192,15.1 \%)$, totaling 3035626 procedures in the period 2008- 2014. There was no
\end{abstract}


statistically significant correlation between the number of procedures and the time of the evaluated period $(p=0.444)$. Regarding the type of management, municipal CEOs, $p=0.003, r=0.964$ and state CEOs, $p=0.048, r=0.775$ ) showed significant growth in the period evaluated. Despite the expansion of secondary oral health services in Ceará and the growing number of registered specialized procedures, the ability to solve the problems of these services is still considered a challenge for oral health managers and professionals.

Keywords: Oral health; Evaluation of health services; Dental specialties.

\section{Resumen}

En Ceará, la expansión de la oferta de los Centros de Especialidades Dentales (CEO) es reciente. Así, el presente estudio tuvo como objetivo evaluar la oferta y producción de los CEO de Ceará en el período de 2008 a 2014 a partir de datos secundarios registrados en el Sistema de Información del Sistema Único de Salud. Los datos se expresaron en forma absoluta y porcentual y la chica utilizó pruebas cuadradas y correlación de Spearman. Los resultados mostraron que el número de procedimientos en 2008 fue de 280222 (9,2\%), con una ligera reducción a $253370(8,3 \%)$ en 2009, un aumento a $677664(22,3 \%)$ en 2010, con posteriores caídas en $2011(\mathrm{n}=525259,17,3 \%), 2012(\mathrm{n}=430991, \mathrm{n}=$ $14,2 \%)$ y $2013(n=291666,9,6 \%)$ retomando el crecimiento en $2014(\mathrm{n}=457192,15,1 \%)$, totalizando 3035626 procedimientos en el período 2008-2014. No hubo correlación estadísticamente significativa entre el número de procedimientos y el tiempo del período evaluado $(\mathrm{p}=0,444)$. En cuanto al tipo de gestión, los directores generales municipales, $\mathrm{p}=0,003, \mathrm{r}=0,964$ y los directores generales estatales, $\mathrm{p}=0,048, \mathrm{r}=0,775$ ) mostraron un crecimiento significativo en el período evaluado. A pesar de la expansión de los servicios secundarios de salud bucal en Ceará y del creciente número de procedimientos especializados registrados, la capacidad de resolver los problemas de estos servicios todavía se considera un desafío para los administradores y profesionales de la salud bucal.

Palabras clave: Salud bucal; Evaluación de servicios de salud; Especialidades dentales.

\section{Introdução}

No Brasil, a atenção odontológica ficou caracterizada, durante anos, por atender grupos populacionais restritos, por meio de programas voltados para o curativismo, tendo como resultado uma baixa cobertura, tanto no aspecto assistencial como populacional (Machado et al., 2015; Oliveira et al., 2005).

A Política Nacional de Saúde Bucal (PNSB), Brasil Sorridente, implantada em 2004, compreende, nos âmbitos individual e coletivo, um conjunto de ações de promoção da saúde, prevenção de agravos, diagnóstico, tratamento e reabilitação (Lino et al., 2014; Ministério da Saúde, 2004). Em linhas gerais, o objetivo maior da PNSB é a reorganização da atenção primária especialmente por meio das Equipes de Saúde Bucal (ESB) na Estratégia de Saúde da Família (ESF). Visa também a ampliação e qualificação da atenção especializada por meio dos Centros de Especialidades Odontológicas (CEO) e Laboratórios Regionais de Prótese Dentária (LRPD) e pela oferta de assistência odontológica na atenção terciária (Ministério da saúde, 2004; Souza et al., 2007).

Neste sentido de qualificação e ampliação da assistência pública odontológica de média complexidade foram instituídos os Centros de Especialidades Odontológicas (CEO) com vista a cumprir o princípio da integralidade da atenção, pois se constatou que o perfil da assistência odontológica no SUS era caracterizado por uma grande desproporção na oferta de procedimentos odontológicos básicos e especializados. Esses são classificados no Cadastro Nacional de Estabelecimentos de Saúde (CNES) como Clínica Especializada ou Ambulatório de Especialidade e são responsáveis pela assistência pública de média complexidade (Machado et al., 2015; Oliveira et al., 2005).

Podem se apresentar em 3 categorias distintas: CEO tipo I (com 3 cadeiras odontológicas), tipo II (com 4 a 6 cadeiras odontológicas) e tipo III (com 7 ou mais cadeiras odontológicas). Os CEOs são um tipo de serviço de saúde que deve oferecer à população, no mínimo os serviços de diagnóstico bucal, com ênfase no diagnóstico e detecção do câncer de boca, periodontia especializada, cirurgia oral menor dos tecidos moles e duro, endodontia e atendimento a pacientes com necessidades especiais (Condessa et al., 2020; Portaria ${ }^{\circ}$ 599, 2006).

O Estado do Ceará tem adotado um modelo fundamentado nos Consórcios de Saúde em sua política de regionalização, compondo a estrutura desses consórcios estão os Centros de Especialidades Odontológicas Regionais (CEOR), sendo implantados nos municípios sede das microrregiões de saúde do Estado de Ceará 8, de acordo com o Plano Diretor 
de Regionalização, dando acesso à população dos municípios adstritos àquela microrregional de saúde. A partir dessa rede assistencial que vem se formando, a prestação pública de serviços em saúde bucal no Ceará tem avançado de um panorama de oferta limitada de procedimentos de baixa complexidade, com reduzida realização de procedimentos especializados, em direção à ampliação do acesso na atenção básica e na média complexidade, tanto no sentido da universalização do acesso quanto da integralidade das ações (Araujo et al., 2012; Couto et al., 2021; Figueiredo et al., 2009; Pinto et al., 2014).

Em vista do papel social dos CEO em reduzir as iniquidades de acesso aos serviços odontológicos especializados e, assim, propiciar reduções na morbidade relacionada à saúde bucal, esses serviços devem ser avaliados em prol da melhoria contínua do seu desempenho. Entretanto, não havia, até então no Brasil, uma política de avaliação de CEO. Recentemente, em 2013, o Governo federal expandiu o Programa de Melhoria do Acesso e Qualidade (PMAQ) aos CEO (PMAQ-CEO) como estratégia de qualificação dos serviços especializados em saúde bucal. O programa avaliará o desempenho dos CEO, e irá repassar mais recursos para os serviços que cumprirem os padrões de qualidade pactuados (Portaria $\mathrm{n}^{\circ}$ 261., 2013; Rosendo et al., 2021).

Como no Brasil, no Ceará, a expansão da oferta de serviços odontológicos especializados é recente. Deste modo, o presente estudo teve como objetivo avaliar a oferta e a produção dos serviços odontológicos especializados do Ceará no período de 2008 a 2014.

\section{Metodologia}

\section{Desenho do estudo, população e amostra}

Este estudo foi do tipo descritivo, quantitativo (Pereira et al., 2018), de caráter avaliativo, o qual utilizou dados secundários, a partir da série histórica da produção de procedimentos odontológicos (quantidade apresentada) realizados nos centros de especialidades odontológicas do Ceará no período de 2008 a 2014.

\section{Local e período do estudo}

Os dados de produção são armazenados nos Sistema de Informações Ambulatoriais do Sistema Único de Saúde (SIA/SUS) de onde foi feita sua coleta, empregando o banco de dados do Departamento de Informática do SUS (DATASUS). As informações foram acessadas em julho de 2015 e a tabulação foi feita por meio do Programa Tab para Windows Programa TABWIN do Ministério da Saúde, sendo exportados para o programa Excel, versão 2010 (Microsoft Corp., Estados Unidos), onde foi feita consolidação e agrupamento de acordo com os grupos de procedimentos odontológicos que deveriam ser avaliados.

\section{Critérios de inclusão}

Todas as unidades de saúde que realizavam procedimentos considerados especializados nas áreas de endodontia, periodontia, cirurgia, prótese e ortodontia pela Política Nacional de Saúde Bucal foram incluídas na análise. Não foram analisados os procedimentos relacionados aos pacientes com necessidades especiais por serem, na maioria das vezes, da atenção primária, sendo difícil distinguir quais foram realizados fora dos Centros de Especialidades Odontológicas. As variáveis relacionadas aos serviços foram: tipo de CEO, que são classificados em função dos seus recursos físico-estruturais, classificados em três tipos: CEO tipo I, CEO Tipo II, e CEO Tipo III; tipo de administração,isto é, se eram CEO's Municipais ou CEO's Estaduais.

\section{Análise dos dados}

Os dados foram exportados ao software GraphPad Prism 5.0 no qual as análises foram realizadas considerando uma confiança de $95 \%$. Os dados foram expressos em forma de frequência absoluta e percentual e utilizaram-se os testes do qui- 
quadrado para avaliação do aumento do número de procedimentos dos CEOS por anos e a correlação não-linear de Spearman para avaliação da tendência de aumento do número dos procedimentos considerando o período avaliado.

\section{Resultados}

O número total de procedimentos no ano de 2008 foi de 280222 (9.2\%), havendo uma leve redução para 253370 (8.3\%) em 2009, um aumento para 677664 (22.3\%) em 2010, com quedas subsequentes em 2011 (n=525259, 17.3\%), 2012 $(\mathrm{n}=430991, \mathrm{n}=14.2 \%)$ e $2013(\mathrm{n}=291666,9.6 \%)$ assumindo crescimento em $2014(\mathrm{n}=457192,15.1 \%)$, totalizando 3035626 procedimentos no período de 2008-2014. Não houve correlação estatisticamente significante do número de procedimentos com o tempo do período avaliado ( $\mathrm{p}=0.444)$ (Tabela 1$)$.

O número de procedimentos periodontais foi o mais representativo nos anos de 2008 ( $\mathrm{n}=194600,11.0 \%$ ), havendo crescimento significante em 2009 ( $\mathrm{n}=176970,10.0 \%)$. Este crescimento foi superado pelos procedimentos endodônticos em $2010(\mathrm{n}=463836,60,5 \%)$, reassumindo a importância em $2011(\mathrm{n}=415911,23,5 \%), 2012(\mathrm{n}=318392,18,0 \%)$ e dando lugar à prótese em 2013 e ortodontia, prótese e cirurgia em 2014 ( $\mathrm{p}<0.001$ ). O número de procedimentos cirúrgicos $(\mathrm{p}=0.003$, $\mathrm{r}=0.964)$, protéticos $(\mathrm{p}=0.012, \mathrm{r}=0.893)$ e ortodônticos ( $\mathrm{p}=0.024, \mathrm{r}=0.857)$ apresentaram crescimento significante ao longo do período avaliado (Figura 1).

Figura 1 - Número de procedimentos por especialidades realizados nos CEO's do estado do Ceará no período de 2008 até 2014.

\begin{tabular}{|c|c|c|c|c|c|c|c|c|c|c|}
\hline & \multicolumn{8}{|c|}{ Ano } & \multirow{2}{*}{$\begin{array}{c}\text { p- } \\
\text { Valo- } \\
\mathbf{r}^{\mathbf{a}} \\
\mathbf{r}\end{array}$} & \multirow{2}{*}{$\begin{array}{l}\text { p- } \\
\text { Va- } \\
\text { lor }\end{array}$} \\
\hline & 2008 & 2009 & 2010 & 2011 & 2012 & 2013 & 2014 & Total & & \\
\hline \multicolumn{11}{|l|}{$\begin{array}{l}\text { Especia- } \\
\text { lidade }\end{array}$} \\
\hline \multirow{2}{*}{$\begin{array}{l}\text { Cirur- } \\
\text { gia }\end{array}$} & 19782 & 20114 & 23002 & 29320 & \multicolumn{2}{|c|}{$3317231704 \quad 136$} & 870 & $\begin{array}{c}30947 \\
3\end{array}$ & \multirow{2}{*}{$\begin{array}{c}* 0.00 \\
3 \\
0.964\end{array}$} & \multirow[t]{2}{*}{$\begin{array}{c}<0.00 \\
1\end{array}$} \\
\hline & $6.4 \%$ & $6.5 \%$ & $7.4 \%$ & $9.5 \%$ & $\begin{array}{c}10.7 \\
\%\end{array}$ & $\begin{array}{c}10.2 \\
\%\end{array}$ & $\begin{array}{c}44.2 \\
\%\end{array}$ & & & \\
\hline \multirow{3}{*}{ Endo } & 5332 & 4525 & 4638 & 5267 & 4790 & 4119 & 4540 & 76701 & \multirow[b]{2}{*}{0.267} & \\
\hline & 1 & 1 & $36 \dagger^{\dagger}$ & 9 & 6 & 6 & 5 & 2 & & \\
\hline & $7.0 \%$ & $5.9 \%$ & $\begin{array}{c}60.5 \\
\%\end{array}$ & $6.9 \%$ & $6.2 \%$ & $5.4 \%$ & $5.9 \%$ & \multirow{4}{*}{$\begin{array}{c}17672 \\
37\end{array}$} & - & \\
\hline \multirow{3}{*}{$\begin{array}{l}\text { Perio- } \\
\text { dontia }\end{array}$} & 1946 & 1769 & 1788 & 4159 & 3183 & 1794 & 2332 & & & \\
\hline & $00^{\dagger}$ & $70^{\dagger}$ & 41 & $11^{\dagger}$ & $92 \dagger$ & 74 & 16 & & 0.396 & \\
\hline & $\begin{array}{l}11.0 \\
\%\end{array}$ & $\begin{array}{l}10.0 \\
\%\end{array}$ & $\begin{array}{c}10.1 \\
\%\end{array}$ & $\begin{array}{c}23.5 \\
\%\end{array}$ & $\begin{array}{c}18.0 \\
\%\end{array}$ & $\begin{array}{c}10.2 \\
\%\end{array}$ & $\begin{array}{c}13.2 \\
\%\end{array}$ & & - & \\
\hline \multirow{4}{*}{ Protese } & 1162 & 1040 & 1130 & 2471 & 2611 & 3074 & 3532 & \multirow{4}{*}{$\begin{array}{c}16456 \\
0\end{array}$} & & \\
\hline & & 5 & 8 & 4 & 7 & 8 & $5 \dagger$ & & ${ }^{*} 0.01$ & \\
\hline & & & & 15.0 & 15.9 & 18.7 & 21.5 & & $\begin{array}{c}2 \\
0.893\end{array}$ & \\
\hline & $7.1 \%$ & $6.3 \%$ & $6.9 \%$ & $\%$ & $\%$ & $\%$ & $\%$ & & & \\
\hline \multirow{2}{*}{$\begin{array}{l}\text { Orto- } \\
\text { dontia }\end{array}$} & 894 & 630 & 677 & 2635 & $5404^{\dagger}$ & $8544 \dagger$ & $6538 \dagger$ & \multirow[t]{2}{*}{$\dagger \quad 27344$} & ${ }_{4} 0.02$ & \\
\hline & $3.3 \%$ & $2.3 \%$ & $2.5 \%$ & $9.6 \%$ & $\begin{array}{c}19.8 \\
\%\end{array}$ & $\begin{array}{c}31.2 \\
\%\end{array}$ & $\begin{array}{c}23.9 \\
\%\end{array}$ & & 0.857 & \\
\hline \multirow{3}{*}{ Total } & 2802 & 2533 & 6776 & 5252 & 4309 & 2916 & 4571 & \multirow{3}{*}{$\begin{array}{l}30356 \\
26\end{array}$} & & \\
\hline & 22 & 70 & 64 & 59 & 91 & 66 & 92 & & 0.444 & \\
\hline & $9.2 \%$ & $8.3 \%$ & $\begin{array}{c}22.3 \\
\%\end{array}$ & $\begin{array}{c}17.3 \\
\%\end{array}$ & $\begin{array}{c}14.2 \\
\%\end{array}$ & $9.6 \%$ & $\begin{array}{c}15.1 \\
\%\end{array}$ & & - & \\
\hline
\end{tabular}


O número de CEOs municipais apresentou grande representatividade nos anos de 2008 ( $\mathrm{n}=39,8.9 \%), 2009$ ( $\mathrm{n}=58$, 13,3\%), 2010 ( $n=66,15,1 \%)$ e 2011 ( $n=68,15,6 \%)$, sendo seu crescimento superado pelo aumento do número de CEOS estaduais em $2012(\mathrm{n}=9,15,8 \%)$ a $2014(\mathrm{n}=20,35,1 \%)(\mathrm{p}<0.001)$. Ambos os CEOS apresentaram crescimento significante no período avaliado (CEO municipais, $\mathrm{p}=0.003, \mathrm{r}=0.964$, $\mathrm{CEO}$ estaduais, $\mathrm{p}=0.048, \mathrm{r}=0.775$ ) (Tabela 2).

O número de CEO tipo I e II apresentou grande representatividade nos anos de 2008 ( $\mathrm{n}_{\text {CEO-I }}=26,9.8 \%$, $\mathrm{n}_{\text {CEO-II }}=13$, $10,8 \%$ ), 2009 ( $\mathrm{n}_{\text {CEO-I }}=36,13.5 \%$, $\mathrm{n}_{\text {CEO-II }}=15,12,5 \%$ ), 2010 (n $\mathrm{n}_{\text {CEO-I }}=42,15.8 \%, \mathrm{n}_{\text {CEO-II }}=16,13,3 \%$ ) e 2011 ( $\mathrm{n}_{\text {CEO-I }}=41,15.4 \%$, $\mathrm{n}_{\text {CEO-II }}=19,15,8 \%$ ), sendo seu crescimento superado pelo aumento significante do número de CEO tipo III entre $2012 \mathrm{e}$ 2014. Apenas o CEO III apresentou crescimento estatisticamente significante no período considerado ( $<<0.001, r=0.994)$ (Figura 2).

Figura 2 - Número de unidades de CEO’s do estado do Ceará no período de 2008 até 2014.

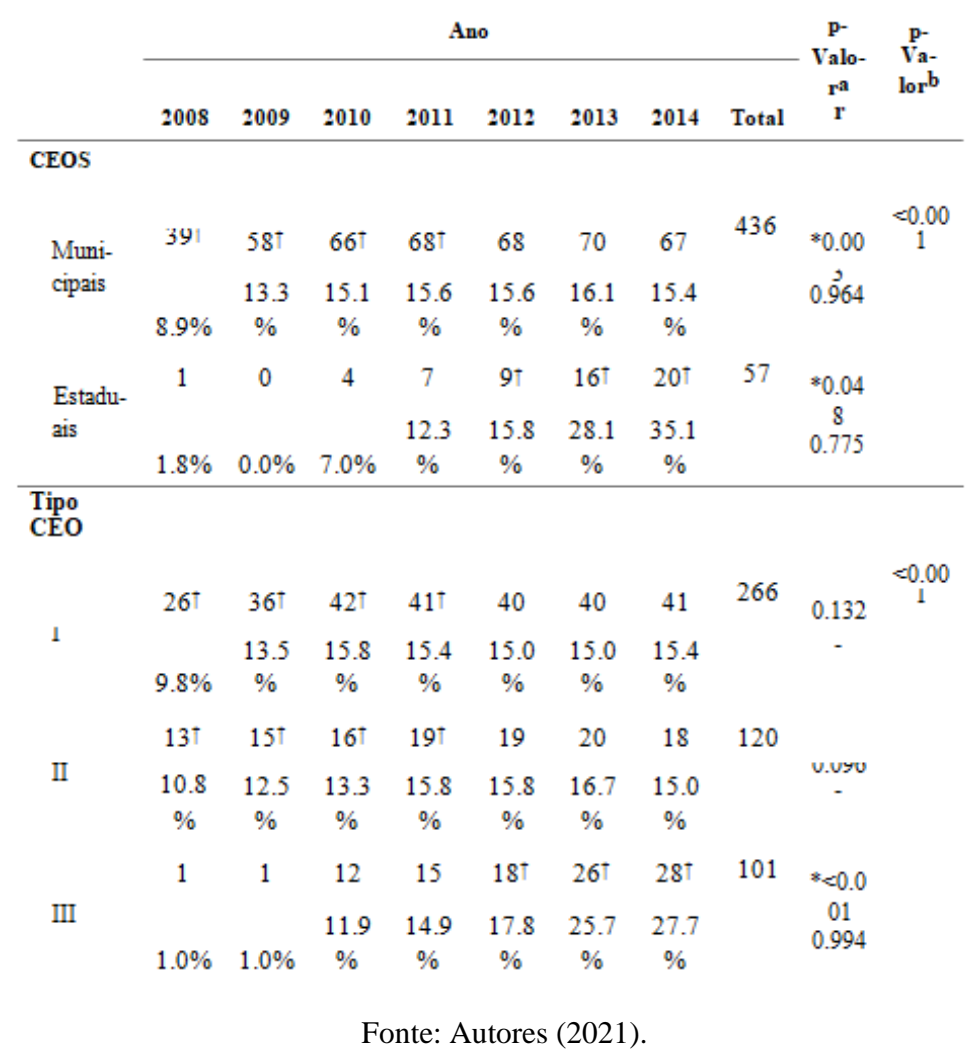

\section{Discussão}

A implantação da Política Nacional de Saúde Bucal (Brasil Sorridente) repercutiu positivamente na ampliação dos serviços de atenção secundária em saúde bucal e, consequentemente, na disponibilização de procedimentos especializados em Odontologia em todo país (Araujo et al., 2012; Araújo et al., 2021; Lima et al., 2010).

De acordo com um estudo realizado em Pernambuco que avaliou os serviços de saúde bucal especializado existentes neste estado, indicou que quanto menor o município ou menor o seu desenvolvimento humano, pior o desempenho dos Centros de Especialidades Odontológicas. Em concordância, ARAUJO et al (2010) mostrou que o estado do Ceará, no período de 2000 a 2010, houve uma considerável aumento do número de CEO, no entanto, a grande maioria sendo do tipo I sob administração municipal, caracterizado por um reduzido número de consultórios odontológicos e por uma produtividade mensal baixa. Uma alternativa a esta situação seria o reforço do Governo Estadual e das Comissões Intergestoras Bipartite Estadual e Regional, tanto no cumprimento do Plano Diretor de Regionalização pactuado, quanto ao 
estímulo à criação de consórcios intermunicipais em saúde para efetivação da regionalização da atenção secundária em saúde bucal evidenciando a importância da implementação de centros regionais de referência odontológica (Araujo et al., 2012; Figueiredo et al., 2009).

Seguindo esta linha de pensamento o Estado do Ceará tem adotado um modelo fundamentado nos Consórcios de Saúde em sua política de regionalização. Compondo a estrutura desses consórcios estão os Centros de Especialidades Odontológicas Regionais (CEO-R) até 2011 doze centros regionais odontológicos especializados foram implementados nos municípios, número ainda insuficiente para assegurar a totalidade dos cuidados de saúde oral para população de todo o estado. No nosso estudo mostramos que no período estudado, principalmente durante os anos de 2012 a 2014, ocorreu um crescimento significativo no número de CEO-R em que atualmente conta com uma rede composta de 22 centros regionais (Araujo et al., 2012; Pinto et al., 2014; Secretaria de saúde do estado, 2008).

Apesar da ampliação da quantidade de CEO o número de procedimentos realizados pelas especialidades pesquisadas cadastrados no SIA-SUS durante o período estudado não apresentou um aumento significativo no Ceará. No estudo de Pinto et al (2013) os CEO-R parecem estar adequadamente preparados para atender à demanda pactuada. Contudo, existem barreiras geográficas (distância e carência de transporte), financeiras (custo do transporte) e organizacionais (falta de dados epidemiológicos para pactuação e oferta de vagas) que dificultam a acessibilidade e melhores taxas de aproveitamento e utilização dos serviços oferecidos nestas unidades. Esta constatação convida a refletir se o simples fato de aumentar a oferta de um serviço especializado é o suficiente para que se tenha uma maior resolutividade. Ser resolutivo no acesso ao sistema significa também promover a oportunidade de utilização dos serviços pelos usuários (Araujo et al., 2012; Pinto et al., 2014; Lino et al., 2014; Machado et al., 2015).

Como estratégia de qualificação dos serviços especializados em saúde bucal e para que se tenha uma maior resolutividade o Governo Federal expandiu o Programa de Melhoria do Acesso e Qualidade (PMAQ) aos CEO (PMAQCEO) e o Ceará é o estado com o maior número de Centros de Especialidades Odontológicas do tipo III certificados com "desempenho muito acima da média". Em todo o país, somente 15 CEO tipo III conquistaram a melhor avaliação de desempenho, seis deles no Ceará, outros seis CEO regionais foram certificados com "desempenho acima da média". Ao todo, foram certificados no Ceará 72 CEO, sendo 17 regionais e 55 municipais, de um total de 76 com adesão ao PMAQCEO (Portaria $\mathrm{n}^{\circ}$ 261, 2013; Portaria No 677, 2015; Rosendo et al., 2020).

\section{Conclusão}

Diante do que foi exposto, observou-se uma ampliação dos serviços de saúde bucal na rede de atenção secundária no estado do Ceará, entretanto a resolutividade desses serviços ainda é considerada um desafio para os gestores e profissionais da assistência em saúde bucal. A deficiência na resolutividade desses serviços pode comprometer o estabelecimento de sistemas de referência e contra-referência em saúde bucal, tendo consequências negativas sobre os princípios doutrinários do SUS como universalidade dos serviços, equidade e integralidade na assistência à saúde.

Outros fatores que contribuirão para aumentar a oferta e a qualidade dos serviços especializados em Odontologia é a manutenção das políticas de saúde bucal, a ampliação dos recursos financeiros destinados para esses serviços, como o incentivo do PMAQ-CEO que foi estabelecido recentemente e a realização de avaliações para subsidiar o planejamento e a tomada de decisão.

Por último, devido à natureza deste estudo, não foi possível analisar em maior profundidade a utilização de procedimentos odontológicos especializados disponibilizados pelos CEO implementados no Ceará para a população do estado. Recomenda-se que novos estudos sejam realizados para avaliar se existem restrições ao acesso a esses serviços, se a produtividade metas estão sendo cumpridas e se o sistema de referência e contra-referência está bem definido. 


\section{Referências}

Araujo, D. B., et al (2012). Atenção secundária em saúde bucal e a implementação dos Centros de Especialidades Odontológicas em um estado do Nordeste, Brasil. Rev Gaucha Odontol, 60(1), 49-54.

Araújo, T. C. L., et al (2021). Absenteeism of patients with special needs in dental specialties centers. Research, Society and Development. 10 , e40310313527.

Brasil. Ministério da saúde (MS). Secretaria de Atenção à Saúde (2004). Departamento de Atenção básica. Coordenação Nacional de Saúde bucal. Diretrizes da Política Nacional de Saúde Bucal.

Brasil. Portaria no 261, de 21 de Fevereiro de 2013 (2013). Institui, no âmbito da Política Nacional de Saúde Bucal, o Programa de Melhoria do Acesso e Qualidade dos Centros de Especialidades Odontológicas (PMAQ-CEO) e o Incentivo Financeiro (PMAQ-CEO), denominado Componente de Qualidade da Atenção Especializada em Saúde Bucal. Diário Oficial da União 2013.

Brasil. Portaria n 5 599, de 23 de Março de 2006 (2006). Define a implantação de Especialidades Odontológicas (CEO) e de Laboratórios Regionais de Próteses Dentárias (LRPDs) e estabelecer critérios, normas e requisitos para seu credenciamento. Diário Oficial da União.

Brasil. Portaria No 677, de 3 de junho de 2015 (2015). Homologa a certificação dos Centros de Especialidades Odontológicas (CEO) no Programa Nacional de Melhoria do Acesso e da Qualidade dos Centros de Especialidades Odontológicas (PMAQ-CEO). Diário Oficial da União.

Ceará. Secretaria de saúde do estado. Plano Estadual de Saúde 2007 - 2010 (2008). Secretaria de Saúde do Estado do Ceará.

Condessa, A. M., et al (2020). Atenção odontológica especializada para pessoas com deficiência no Brasil: perfil dos centros de especialidades odontológicas, 2014. Epidemiologia e servicos de saude: revista do Sistema Unico de Saude do Brasil, 29(5), e2018154.

Couto, G. R., et al (2021). Analysis of performance of specialized dental care in a care network for people with special needs. Research, Society and Development. 10, e35710212678.

Figueiredo, N., et al (2009). Construção da atenção secundária em saúde bucal: um estudo sobre os Centros de Especialidades Odontológicas em Pernambuco, Brasil. Cad. Saúde Pública, 25(2), 259-267.

Lima, A. C. S., et al (2010). Satisfação dos usuários assistidos nos Centros de Especialidades Odontológicas do Município do Recife, Pernambuco, Brasil. Cad Saude Publica, 26(5), 991-1002.

Lino, P. A., et al (2014). Análise da atenção secundária em saúde bucal no estado de Minas Gerais, Brasil. Cien Saude Colet , 19(9), 3879-3888.

Machado, et al (2015). Fatores relacionados ao desempenho de Centros de Especialidades Odontológicas. Cien Saude Colet, 20(4), 1149-1163.

Oliveira, et al (2005). Atenção odontológica no Programa de Saúde da Família de Campos dos Goytacazes. Cien Saude Colet, 10(1), $297-302$.

Pereira A. S., et al. (2018). Metodologia da pesquisa científica. UFSM. https://repositorio.ufsm.br/bitstream/handle/1/1582 4/Lic_Computacao_Metodologia-Pesquisa-Cientifica.pdf?sequence=1

Pinto, V. P. T., et al (2014). Avaliação da acessibilidade ao Centro de Especialidades Odontológicas de abrangência macrorregional de Sobral, Ceará, Brasil. Cien Saude Colet, 19(7), 2235-2244.

Rosendo, C. de P., et al (2021). Prevalência de necessidades especiais de pacientes atendidos em um CEO tipo III de uma referência hospital no Brasil. Pesquisa, Sociedade e Desenvolvimento. 10, e58510112097.

Rosendo, R. A., et al (2020). Degree of satisfaction of users of a Dental Specialties Center in Paraíba. Research, Society and Development. 9 , e167985502.

Santana, V. G. D., et al (2008). Análise da evolução e financiamento da assistência odontológica na média complexidade no município do Recife no período de 2000 a 2007. Cad. Saúde Colet, 6(3), 527-544.

Souza, T. M. S., et al (2007). Saúde bucal no Programa de Saúde da Família: uma avaliação do modelo assistencial. Cad Saude Publica, 23(11), 27272739 . 